\title{
The Evaluation of Short- and Long-Term Performance of Cold-Mix Asphalt Patching Materials
}

\author{
Chien-Wei Huang (iD, Tsung-Han Yang, and Guan-Bo Lin \\ Department of Civil Engineering, National Chung Hsing University, Taichung 40227, Taiwan \\ Correspondence should be addressed to Chien-Wei Huang; stanhuang917@nchu.edu.tw
}

Received 26 February 2020; Revised 24 May 2020; Accepted 8 June 2020; Published 23 July 2020

Academic Editor: Hossein Moayedi

Copyright (C) 2020 Chien-Wei Huang et al. This is an open access article distributed under the Creative Commons Attribution License, which permits unrestricted use, distribution, and reproduction in any medium, provided the original work is properly cited.

\begin{abstract}
Potholes, one of the major types of distress on pavement surfaces, damage vehicles and are a safety hazard for the travelling public. In order to mitigate the effect of potholes, cold-mix asphalt (CMA) patching materials are commonly used for urgent repair of pavement surfaces before resurfacing can be undertaken. Therefore, the short-term (initial stability) and long-term (in-service durability) performance evaluation of CMA patching materials is necessary. This study conducted several curing conditions in the laboratory to investigate short-term, long-term, and moisture effects on pavement surfaces. Moreover, this study compared the Marshall stability of samples prepared under various compaction conditions. Marshall stability, Cantabro abrasion, and UK wheel tracking tests were conducted to evaluate the performance of CMA patching materials. The results indicated that the Marshall stability of dense-gradation (DG) CMA patching materials was higher than that of open-graded (OG) CMA patching materials and the Marshall stability of OG CMA patching materials was highly influenced by the coarse aggregate proportion. The DG and OG CMA patching materials exhibited comparable abrasion resistance, and the Cantabro abrasion ratio was highly correlated to the estimated asphalt film thickness for the OG CMA patching materials. A moisture indicator (MI) was proposed, and the effect of moisture damage on Marshall stability and Cantabro abrasion ratio was related to the proposed MI. The rutting resistance of the DG CMA patching materials was higher than that of the OG CMA patching materials, which is consistent with the Marshall stability result.
\end{abstract}

\section{Introduction}

Pothole distress considerably degrades pavement performance and affects safety and driving quality. However, pavement resurfacing is expensive and time consuming and requires considerable human resources. Cold-mix asphalt (CMA) patching materials can be easily and swiftly prepared for repairing potholes on a pavement surface. Moreover, the construction and compaction of CMA patching material can be conducted at ambient temperatures, which considerably reduces energy requirements [1]. Therefore, CMA patching materials are efficient, time saving, and economical for pavement maintenance. CMA patching materials are produced by mixing aggregate with liquid asphalt binders such as cutback asphalts and emulsified asphalts. To ensure satisfactory workability of CMA patching materials at ambient temperatures, cutback asphalts are manufactured by adding petroleum solvents to asphalt binders; emulsified asphalts are produced by mixing an asphalt binder, water, and an emulsifying agent. A low-viscosity-grade asphalt binder is generally selected for cold weather, whereas a highviscosity-grade asphalt binder is considered for warm weather conditions. In some cases, a modified asphalt binder is used to produce the liquid asphalt binder for better durability. The performance of CMA patching materials is related to the resistance of the material to rutting, abrasion, and moisture damage [2]. Rutting refers to the cumulative permanent deformation of asphalt materials [3]. Abrasion is induced by insufficient bonding between aggregates within the asphalt mixtures [4]. Moisture damage is caused by water penetrating into the asphalt mixture, and it degrades the durability of asphalt mixtures $[5,6]$. 
Various laboratory tests, such as the Marshall stability, Cantabro, and wheel tracking tests, are used to evaluate the performance of CMA patching materials. Generally, opengraded (OG) CMA patching materials contain high air voids, which reduce deformation resistance, whereas densegraded (DG) CMA patching materials provide superior deformation resistance [7, 8]. Rezaei et al. [9] performed the Marshall stability, indirect tensile, and Hamburg wheel tracking tests to evaluate the rutting and moisture damage resistance of nine ready-to-use CMA patching materials. Marshall stability and indirect tensile strength tests were performed by curing CMA patching materials overnight at $135^{\circ} \mathrm{C}$ and then compacting the specimens by applying 75 blows on each side of the sample using a Marshall hammer. Furthermore, some specimens of the CMA patching materials were cured at $25^{\circ} \mathrm{C}$ for $24 \mathrm{~h}$ to evaluate the early-life rutting potential. Another sample set was cured at $135^{\circ} \mathrm{C}$ for $18 \mathrm{~h}$ to investigate the effects of curing on rutting susceptibility. The results indicated that DG CMA patching materials exhibited higher resistance to permanent deformation than OG CMA patching materials and that the proportion of coarse aggregates affected the moisture damage and rutting resistances of OG CMA patching materials. Liao et al. [10] performed Marshall stability and indirect tensile tests to study the strength of CMA patching materials and developed a simplified wheel track test to evaluate the moisture damage resistance of CMA patching materials. Compaction was conducted by applying 50 blows on each side at $110^{\circ} \mathrm{C}$, which was followed by curing at $110^{\circ} \mathrm{C}$ for $24 \mathrm{~h}$. Subsequently, 25 blows were applied on each side at $110^{\circ} \mathrm{C}$. The results indicated that the strength and rutting resistance of the highviscosity CMA patching material were comparable with those of hot-mix asphalt (HMA) and that a viscosity of 130 poise at $60^{\circ} \mathrm{C}$ can be set as a desirable engineering property of CMA patching materials. Chen et al. [11] conducted Marshall, indirect tensile, and wheel tracking tests to study the engineering properties of CMA patching materials. In the study, the samples of CMA patching materials were cured at $20^{\circ} \mathrm{C}$ for 1 day to simulate the initial stability. Furthermore, the samples of CMA patching materials were cured at $60^{\circ} \mathrm{C}$ for 3,7 , and 14 days to simulate in-service durability. The results indicated that the curing time, nominal maximum aggregate size, curing temperature, and binder type affected the performance of CMA patching materials. Ling et al. [7] studied the effect of aggregate coatings on the moisture susceptibility of CMA patching materials by conditioning the samples in a $25^{\circ} \mathrm{C}$ water bath for 1 day as well as in an environmental chamber maintained at $30^{\circ} \mathrm{C}$ to evaluate CMA patching materials with and without moisture damage. The tensile strength ratio test indicated that CMA patching materials with a higher level of coating exhibited higher resistance to moisture damage. Minegishi et al. [12] investigated the performance of CMA patching materials and formulated the performance requirements for CMA patching materials. The study performed Marshall, wheel tracking, tensile, and Cantabro abrasion tests under various curing conditions to examine the initial stability and inservice durability of CMA patching materials. Samples were cured at $20^{\circ} \mathrm{C}$ for 1 day to evaluate initial stability; furthermore, samples were cured at $60^{\circ} \mathrm{C}$ for 7 days to simulate in-service durability. Munyagi [8] studied the performance and mechanical properties of five CMA patching materials by evaluating volumetric properties, permeability, indirect tensile strength, and accelerated pavement tests. Compaction was conducted at an ambient temperature to represent actual field compaction conditions. The samples were cured at 30 and $40^{\circ} \mathrm{C}$ for 3 days to simulate short-term and in-service durability, respectively. AlBusaltan et al. [13] calculated the indirect tensile stiffness modulus and creep stiffness through indirect tensile stiffness modulus and uniaxial compressive cyclic tests, respectively, to characterize the mechanical properties of CMA patching materials. The samples were compacted at an ambient temperature and cured at $20^{\circ} \mathrm{C}$ for $24 \mathrm{~h}$. Then, the samples were cured at 20,40 , and $60^{\circ} \mathrm{C}$ and tested at $2,7,14,28,90$, and 180 days. Diaz [14] evaluated the performance of CMA patching materials by performing modified cyclic creep and light cone penetrometer tests. Estakhir and Button [15] conducted unconfined compression tests to evaluate the workability of CMA patching materials. Rosales-Herrera et al. [16] conducted slump and Hamburg wheel tracking tests to evaluate the workability and stability of locally produced CMA patching materials.

Although many tests have been developed to evaluate the performance and mechanical properties of CMA patching materials, most studies have focused on workability and short-term performance; furthermore, although CMA patching materials can be used as a permanent solution for pavement maintenance [14], the evaluation methods and indicators related to the performance of CMA patching materials are unclear [9]. Therefore, the investigation of indicators related to the short- and long-term performance of CMA patching materials is essential.

\section{Research Objectives}

The main objective of this study was to investigate and evaluate the indicators that correlate to Marshall stability and resistance to moisture damage, abrasion, and rutting for short- and long-term performance of CMA patching materials. Four curing processes, motivated by the literature, were conducted to simulate short-term, long-term, and moisture conditions. Moreover, this study examined the effect of sample fabrication conditions by comparing three compaction methods summarized from the literature. Marshall stability, Cantabro abrasion, and UK wheel tracking tests were performed to evaluate the short- and long-term performance of CMA patching materials.

\section{Materials}

Six ready-to-use CMA patching materials (CMA-1 to CMA6), which are widely used and commercially available in Taiwan, were studied. To characterize the physical properties of the CMA patching materials, each CMA patching material was subjected to binder extraction and aggregate gradation tests according to AASHTO-T164 [17] and AASHTO-T30 [18], respectively. Figure 1 presents the 


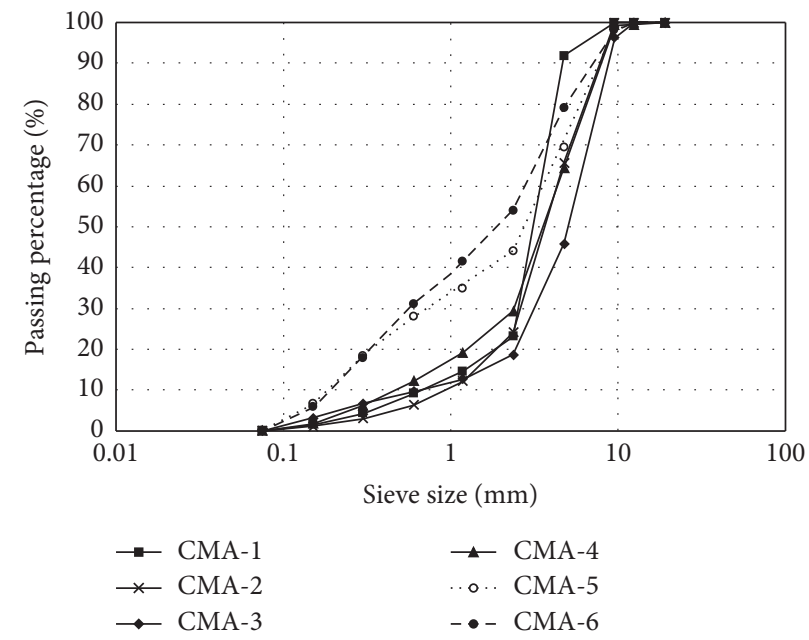

FIGURE 1: Aggregate gradation of the collected CMA patching materials.

TABLE 1: Residual asphalt content in the collected CMA patching materials.

\begin{tabular}{lcccccc}
\hline CMA & CMA-1 & CMA-2 & CMA-3 & CMA-4 & CMA-5 & CMA-6 \\
\hline Asphalt content (\%) & 5.76 & 4.28 & 4.14 & 4.70 & 4.52 & 4.62 \\
\hline
\end{tabular}

results of aggregate gradation; CMA-5 and CMA-6 were DG, whereas CMA-1 to CMA-4 were OG. Among OG aggregate gradations, CMA-1 contained the lowest amount of coarse aggregates, whereas CMA-3 possessed the highest amount of coarse aggregates. The coarse aggregate distribution was similar in CMA-2 and CMA-4, but CMA-4 contained more fine aggregate than CMA-2. Regarding DG aggregate distributions, CMA-5 contained more coarse aggregate than CMA-6, but CMA-5 and CMA-6 exhibited similar fine aggregate content.

Table 1 presents the residual asphalt content for each CMA patching material. Among OG CMA patching materials, CMA-1 exhibited the highest asphalt content (5.76\%), and CMA-3 exhibited the lowest (4.14\%). Among DG CMA patching materials, CMA-5 and CMA- 6 exhibited similar asphalt content. In this study, the asphalt grade was AC-20 for all collected CMA patching materials.

3.1. Sample Fabrication and Preparation. The specimens of CMA patching material were compacted using the Marshall compactor to a height and diameter of $63.5 \pm 5.1 \mathrm{~mm}$ and $101.6 \mathrm{~mm}$, respectively. To study the effect of sample fabrication conditions on CMA patching materials, three compaction processes (labeled A, B, and C)-designed by considering the compaction conditions reported in the literature-were evaluated. In process $A$, the specimens were compacted by applying 75 blows on each side at an ambient temperature. This compaction simulates field compaction at an ambient temperature $[8,13]$. In process $B, 50$ blows were applied on each side at an ambient temperature, and then the specimens were cured at $110^{\circ} \mathrm{C}$ for 1 day. Subsequently, the specimens were compacted with 25 additional blows on each side at $110^{\circ} \mathrm{C}$ [19]. In process C, standard Marshall sample fabrication was followed for preparing HMA. In this method, the CMA patching materials were heated overnight at $135^{\circ} \mathrm{C}$. Next, 75 blows were applied on each side of the specimen [9]. For each compaction method, the air void content of the specimen was maintained within $10 \pm 1 \%$.

To investigate the short-term (initial stability) and longterm (in-service durability) performance of CMA patching materials, four curing processes ( $\mathrm{C} 1$ to $\mathrm{C} 4$ ) - designed by considering the curing conditions reported in the literature-were used $[9,11,12]$. C1 and C2 were designed to evaluate short-term performance. In $\mathrm{C} 1$, the specimens were cured at an ambient temperature $\left(25^{\circ} \mathrm{C}\right)$ for 1 day; in $\mathrm{C} 2$, the specimens were cured at $25^{\circ} \mathrm{C}$ for 1 day, and then the specimens were immersed in a water bath at $60^{\circ} \mathrm{C}$ for 2 days followed by curing at an ambient temperature of $25^{\circ} \mathrm{C}$ for 1 day. The test results of processes $\mathrm{C} 1$ and $\mathrm{C} 2$ can reflect the short-term resistance of CMA patching materials to moisture damage. Processes C3 and C4 were designed to evaluate the long-term performance of CMA patching materials. In $\mathrm{C} 3$, the specimens were oven-cured at $60^{\circ} \mathrm{C}$ for 7 days followed by curing at an ambient temperature $\left(25^{\circ} \mathrm{C}\right)$ for 1 day. In $\mathrm{C} 4$, the specimens were first cured in an oven at $60^{\circ} \mathrm{C}$ for 7 days. Then, the samples were placed in a water bath at $60^{\circ} \mathrm{C}$ for 2 days, after which the samples were cured for 1 day at an ambient temperature $\left(25^{\circ} \mathrm{C}\right)$. The $\mathrm{C} 3$ and $\mathrm{C} 4$ test results indicated the long-term resistance of CMA patching materials to moisture damage. Sample fabrication methods $\mathrm{A}-\mathrm{C}$ and curing processes $\mathrm{C} 1-\mathrm{C} 4$ were combined, as depicted in Figure 2, to comprehensively investigate the short- and long-term performance of the CMA patching materials. Five replicates of each CMA patching material were prepared for every test. If the tendencies of the test results were inconsistent for some replicates, the replicates were discarded, and new replicates were prepared for 


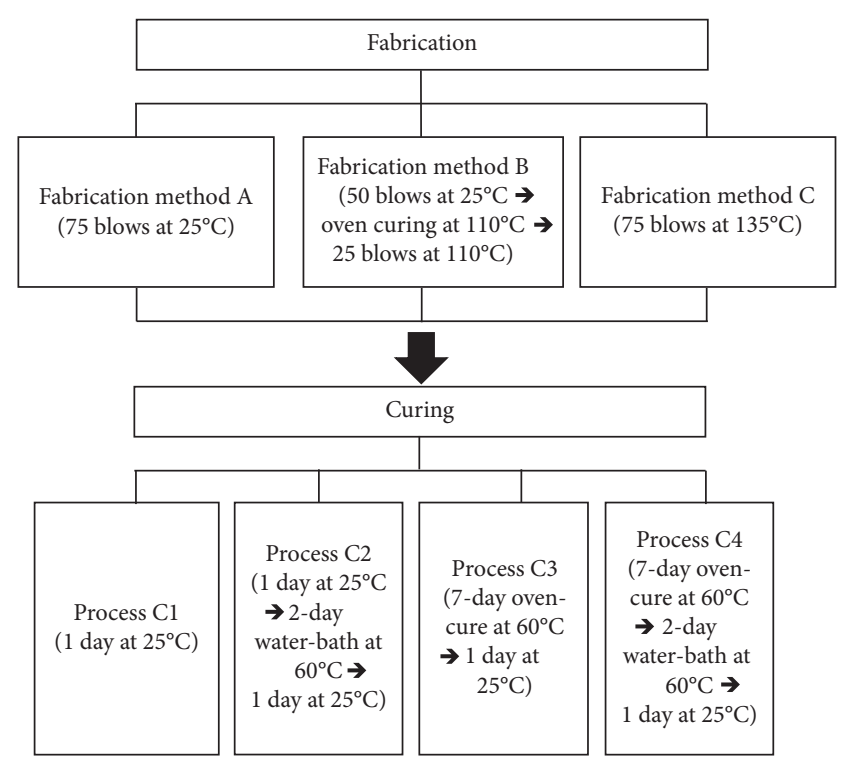

Figure 2: Flowchart of sample fabrication and the curing process.

retesting. The results in the following sections are the mean values of valid replications.

\section{Test Methods}

Marshall stability, Cantabro, and UK wheel tracking tests were conducted to evaluate the performance of CMA patching materials. The Marshall stability test was mainly used to evaluate the short- and long-term strength and moisture susceptibility of CMA patching materials. This test was also conducted to investigate the effect of sample fabrication. The abrasion resistance and the effect of moisture damage on abrasion resistance for short- and long-term performance were evaluated by the Cantabro test. The UK wheel tracking test was then conducted for investigating resistance to rutting.

4.1. Marshall Stability Test. The Marshall stability test was performed in accordance with ASTM D6927 [20]. The cured specimen was placed on a Marshall test machine, and a load was applied on the side of the specimen at a rate of $50.8 \mathrm{~mm} /$ min. Marshall stability is determined as the peak load from the load-deformation curve. The test was conducted at an ambient temperature $\left(25^{\circ} \mathrm{C}\right)$; the sample fabrication and curing conditions are depicted in Figure 2.

4.2. Cantabro Test. The Cantabro test has been widely used to evaluate the abrasion resistance of asphalt mixtures in Europe for many years. In this study, the Cantabro test was conducted according to ASTM D7064 [21]. The mass of the specimen was first measured, and the specimen was then placed in the Cantabro machine without steel balls. The machine was operated for 300 revolutions with 30 to 33 revolutions/min at an ambient temperature $\left(25^{\circ} \mathrm{C}\right)$. The tested specimen was then removed from the machine and the residual mass was measured. The abrasion loss (\%) was determined using the following expression:

$$
R_{a}=\frac{M_{1}-M_{2}}{M_{1}} * 100,
$$

where $M_{1}$ is the mass of specimen before testing, $M_{2}$ is the measured mass of the specimen after testing, and $R_{a}$ is the abrasion loss (\%).

The Cantabro samples were compacted at an ambient temperature, as depicted in Figure 2 (group A) and cured following processes $\mathrm{C} 1$ to $\mathrm{C} 4$. The test results obtained for samples subjected to $\mathrm{C} 1$ and $\mathrm{C} 2$ (C3 and $\mathrm{C} 4$ ) revealed the effect of short-term (long-term) moisture damage on abrasion resistance.

4.3. Rutting Test. In this study, the UK wheel tracking test-a test widely used to evaluate the rutting resistance of CMA patching materials-was performed according to the EN12697-22 specifications [22]. A slab compactor was used to fabricate a $300 \times 300 \times 50 \mathrm{~mm}^{3}$ slab at an ambient temperature $\left(25^{\circ} \mathrm{C}\right)$, thus simulating field compaction. A rubber wheel was repeatedly rolled on the slab 21 times per minute. The rut depth was recorded after each roll.

\section{Results and Discussion}

5.1. Marshall Stability. Figures 3(a) and 3(b) display the Marshall stability results for the various sample fabrications subjected to short-term (C1) and long-term (C3) curing, respectively. The sample fabrication method $\mathrm{C}$ yielded the highest Marshall stability, whereas method A yielded the lowest. However, field compaction is conducted at an ambient temperature, which is similar to fabrication condition A. Therefore, the compaction of CMA patching materials under heating (fabrication conditions $\mathrm{B}$ and $\mathrm{C}$ ) in the laboratory resulted in overestimation of the field strength of the CMA patching materials. Overall, the Marshall stability of DG CMA patching materials (CMA-5 and CMA-6) was higher than that of OG CMA patching materials (CMA-1 to CMA-4) under both short- and long-term curing. The results demonstrated that DG CMA patching materials exhibited higher stiffness than OG CMA patching materials, which was consistent with the results of Rezaei et al. [9].

According to previous studies, the Marshall stability of the OG CMA patching material is related to the proportion of coarse aggregate. Figures 4(a) and 4(b) present the correlation between the Marshall stability and proportion of the coarse aggregate (remaining on the \#4 sieve) in OG patching materials subjected to short- and long-term curing, respectively. The coefficient of correlation was 0.95 and 0.86 for samples fabricated using methods B and C, respectively, which indicated that Marshall stability has a high correlation with the proportion of coarse aggregate. However, the correlation was unsatisfactory for sample fabrication method A because the method A sample was compacted at the ambient temperature and the curing process was not sufficiently long to gain adequate strength. Therefore, Marshall stability is not directly related to the proportion of 


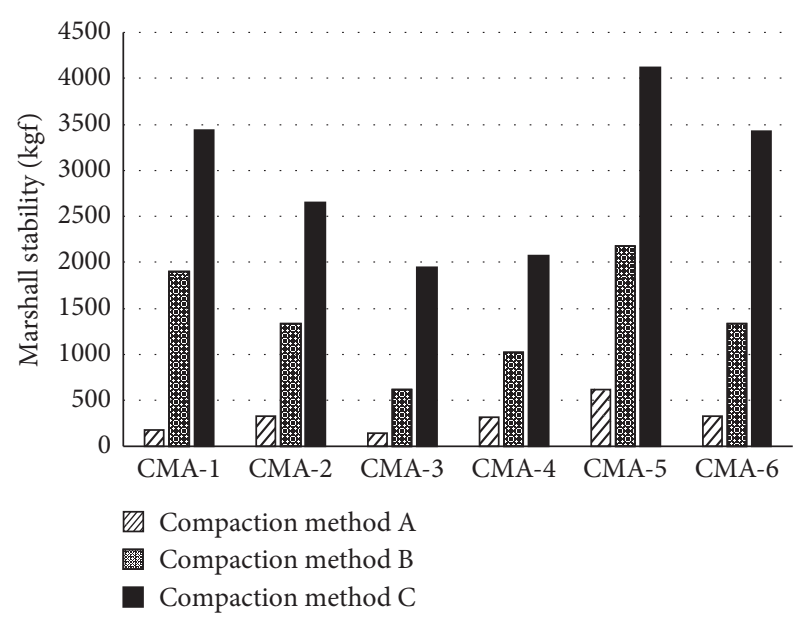

(a)

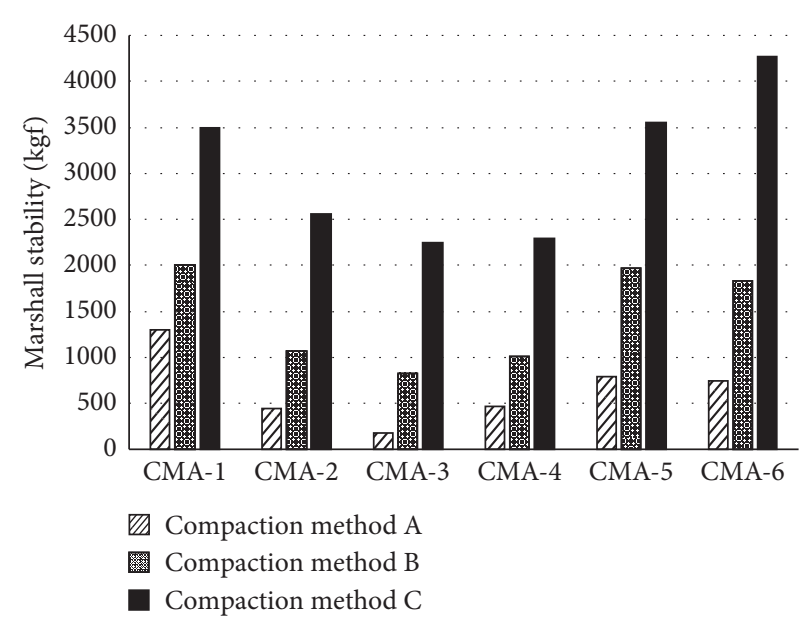

(b)

FIGURE 3: Marshall stability indicating (a) short- and (b) long-term performance.

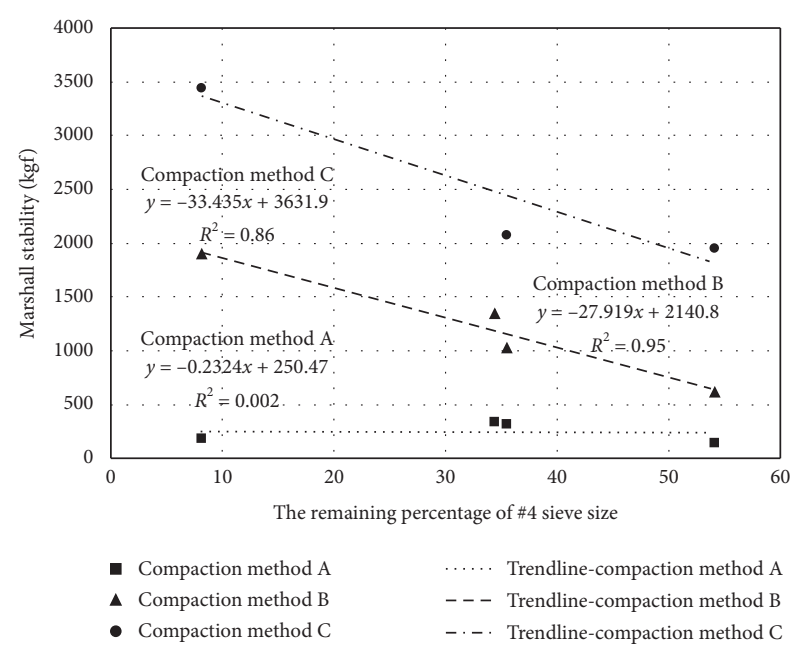

(a)

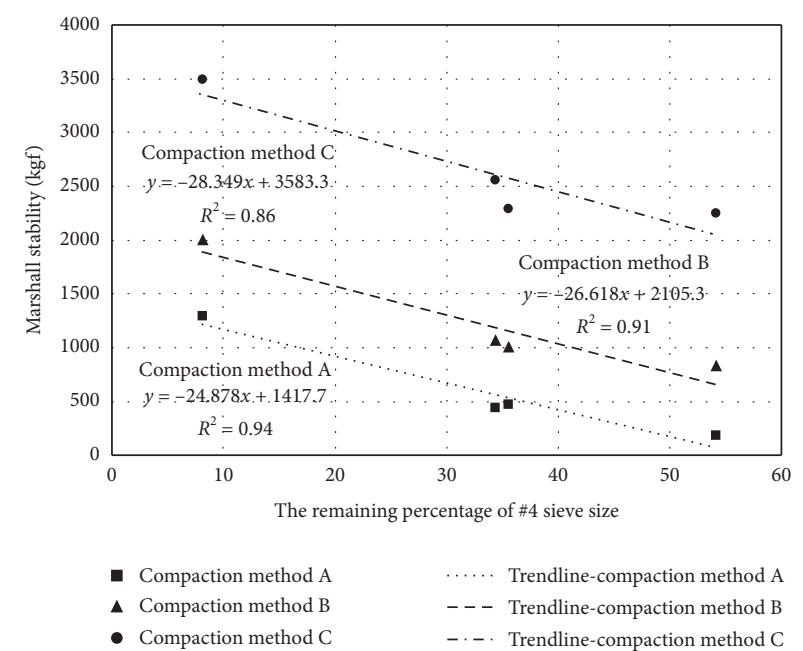

(b)

FIgURE 4: Correlation between Marshall stability and proportion of coarse aggregate indicating (a) short- and (b) long-term performance.

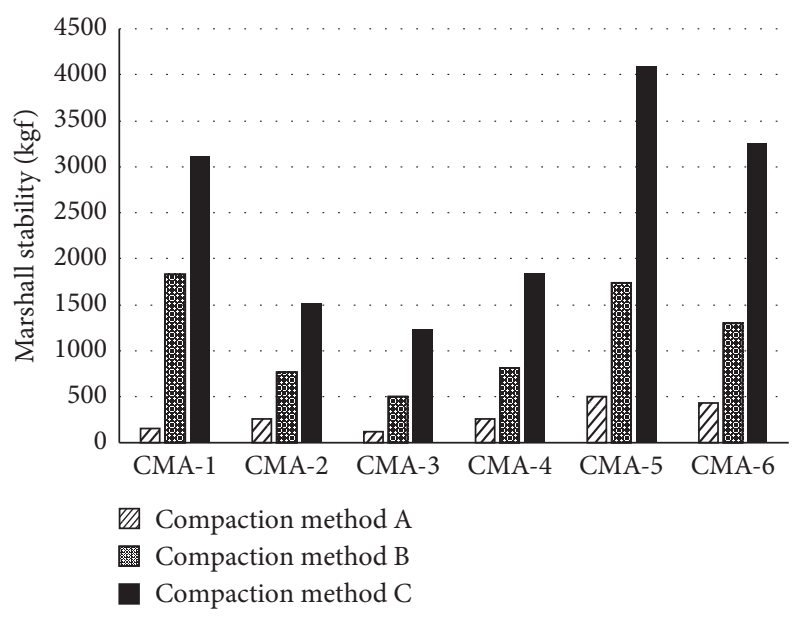

(a)

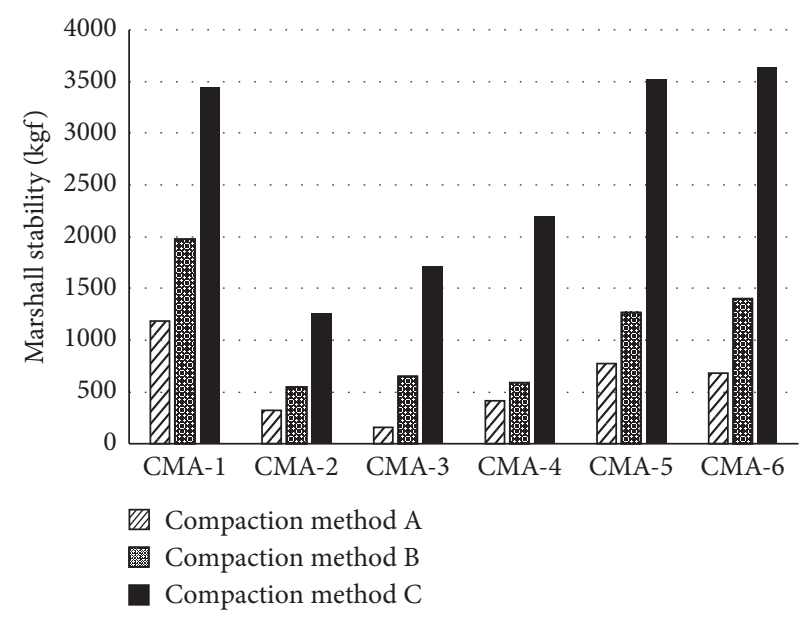

(b)

FIgURE 5: Marshall stability of the sample with moisture damage for (a) short- and (b) long-term performance. 
the coarse aggregate. Regarding the correlation between Marshall stability and the proportion of coarse aggregate in long-term performance, Figure 4(b) indicates that the coefficients of correlation were $0.94,0.91$, and 0.86 for sample fabrication methods $\mathrm{A}, \mathrm{B}$, and $\mathrm{C}$, respectively; these results indicate that Marshall stability is highly correlated to the proportion of the coarse aggregate. Therefore, the proportion of the coarse aggregate can be used as an indicator of Marshall stability.

\subsection{Moisture Damage Evaluation for Marshall Stability} (Residual Marshall Stability). Figures 5(a) and 5(b) present the Marshall stability results of the samples for short-term (C2) and long-term (C4) performance with the moisture condition, respectively. The figure shows that sample fabrication method $\mathrm{C}$ yielded the highest Marshall stability, whereas method A exhibited the lowest Marshall stability. The results are consistent with the Marshall stability of shortterm and long-term performance without the moisture condition.

Residual Marshall stability (RMS) was used to evaluate the moisture damage resistance of CMA patching materials. RMS is defined as the ratio of Marshall stability with moisture damage to Marshall stability without moisture damage, as presented in the following equation:

$$
\mathrm{RMS}=\frac{\mathrm{MS}_{m}}{\mathrm{MS}_{d}} * 100
$$

where $\mathrm{MS}_{m}$ is the Marshall stability with moisture damage (e.g., after curing processes $\mathrm{C} 2$ and C4) and $\mathrm{MS}_{d}$ is the Marshall stability without moisture damage (e.g., after curing processes $\mathrm{C} 1$ and $\mathrm{C} 3$ ). Higher RMS represents lower moisture susceptibility. Short-term RMS was calculated using the ratio of Marshall stability between C2 and C1, and the ratio of Marshall stability between C4 and C3 was used to calculate long-term RMS. Short- and long-term RMS indicate the initial and in-service resistance to moisture damage, respectively.

Figures 6(a) and 6(b) display the RMS results regarding resistance to short- and long-term moisture damage, respectively. In Figure 6, the results of RMS were not related to sample fabrication methods. Figure 7 illustrates the curing and moisture damage effects on Marshall stability during the moisture condition process, revealing that two actions occur simultaneously in CMA patching materials: (a) curing, which increases the strength of CMA patching materials, and (b) moisture damage, which reduces the strength of CMA patching materials. If the increased strength is close to decreased strength, as shown in Figure 7(a), then the CMA patching material exhibits a high RMS. When the difference between increased and decreased strength is significant (Figure $7(\mathrm{~b})$ ), then the CMA patching material exhibits low RMS. Therefore, RMS depends on the relationship between the rate of the increase in strength due to curing and the rate of decreased strength due to moisture damage. However, each compaction method has different potentials for curing and moisture damage, which are related to the material properties. Therefore, RMS does not necessarily have a direct relationship with sample fabrication methods.

Overall, the DG CMA patching materials did not exhibit superior ability to resist moisture damage. Among OG CMA patching materials, CMA-1 exhibited the highest RMS, whereas CMA-2 exhibited the lowest for both short- and long-term performance under all sample fabrication methods. These results indicated that CMA-1 has high moisture resistance and that CMA-2 is susceptible to moisture damage. According to [9], the resistance of OG CMA patching material to moisture damage is associated with aggregate proportion. This study proposed the moisture indicator (MI) for indicating the moisture damage resistance of OG CMA patching material.

$$
\mathrm{MI}=\frac{P_{\# 4}}{P_{\# 50}} .
$$

This indicator is the ratio of the percentage of the aggregate remaining on the $\# 4$ sieve $\left(P_{\# 4}\right)$ to the percentage of aggregate passing through the $\# 50$ sieve $\left(P_{\# 50}\right)$. Thus, this indicator represents the ratio of coarse aggregate to fine aggregate. Figures 8(a) and 8(b) display the correlation between the RMS and MI for short- and long-term performance, respectively. The results revealed that a higher MI indicates higher susceptibility to moisture damage; this is because a higher MI indicates that the mixture contains more open gradation, which allows water to easily penetrate into the mixture. The coefficient of correlation for shortterm performance was $0.87,0.89$, and 0.82 for sample fabrication methods $\mathrm{A}, \mathrm{B}$, and $\mathrm{C}$, respectively. Regarding long-term performance, the coefficient of correlation was $0.86,0.64$, and 0.86 for methods $\mathrm{A}, \mathrm{B}$, and $\mathrm{C}$, respectively. The results demonstrated that the proposed MI was correlated with the moisture damage resistance of OG CMA patching materials.

However, the ability of asphalt mixtures to resist moisture damage is not related only to the aggregate proportion but is affected by many factors [23]. The surface properties of aggregates, such as hydrophilic and hydrophobic properties, are critical in the moisture sensitivity of asphalt mixtures [23-25]. The adhesion between aggregate and asphalt binder considerably affects the moisture resistance of asphalt mixtures, and adhesion is related to the interaction of surface free energy between the asphalt binder and aggregate $[26,27]$. Moreover, the asphalt mixture design parameters, such as air void level, asphalt film thickness, and surface area of aggregates, influence the moisture susceptibility of asphalt mixtures [23, 28, 29]. The proposed moisture damage indicator in this research is a preliminary indicator, in which only the effect of the aggregate proportion is considered. A moisture damage indicator considering more influential factors on the resistance of CMA patching materials to moisture damage is desired.

5.3. Cantabro Test: Abrasion. Figure 9 depicts the Cantabro abrasion ratios for short- and long-term performance without moisture damage (i.e., curing processes $\mathrm{C} 1$ and $\mathrm{C} 3$ ). 


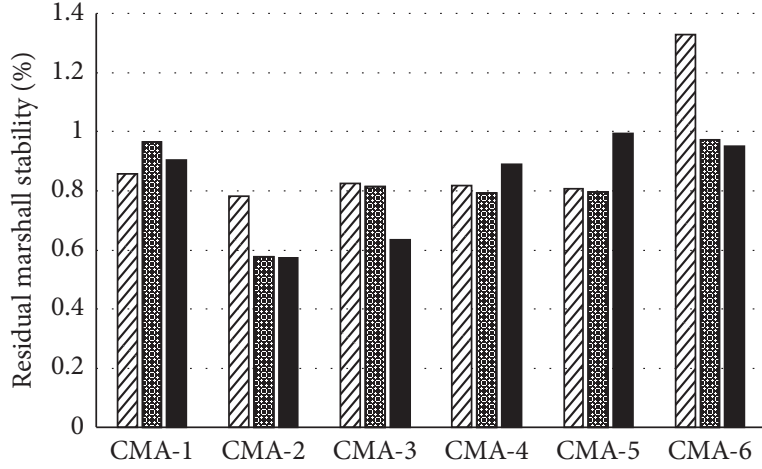

Compaction method A

娄 Compaction method B

- Compaction method C

(a)

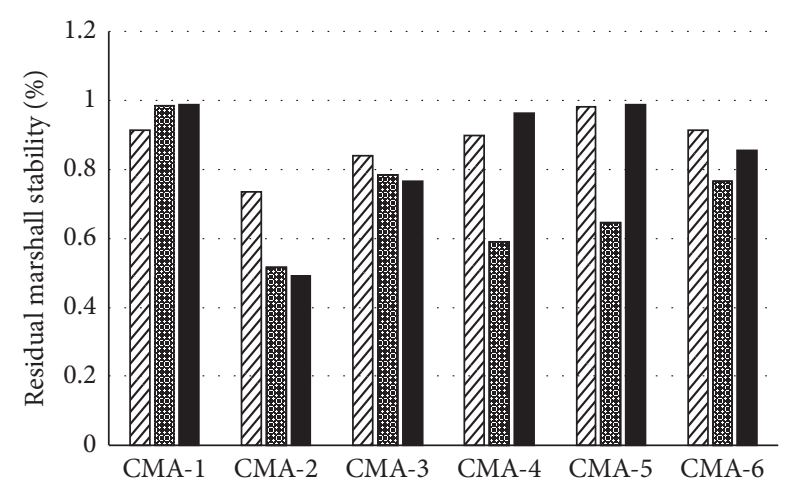

Compaction method A

国 Compaction method B

- Compaction method $\mathrm{C}$

(b)

FIgURE 6: RMS for (a) short- and (b) long-term performance.

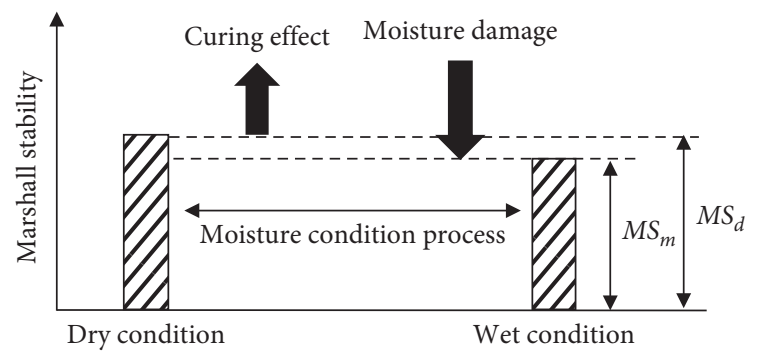

(a)

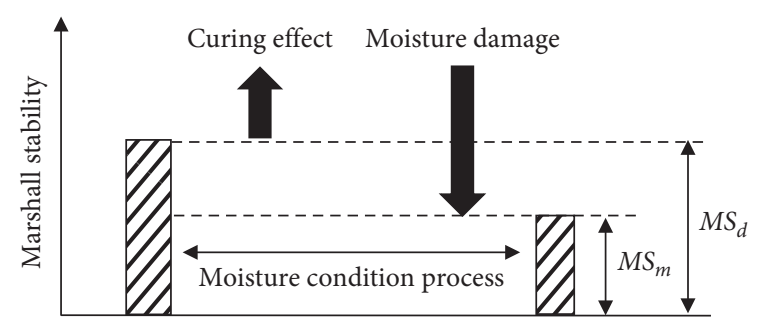

Wet condition

(b)

FIGURE 7: Schematic of the curing and moisture damage effect on Marshall stability during the moisture condition process.

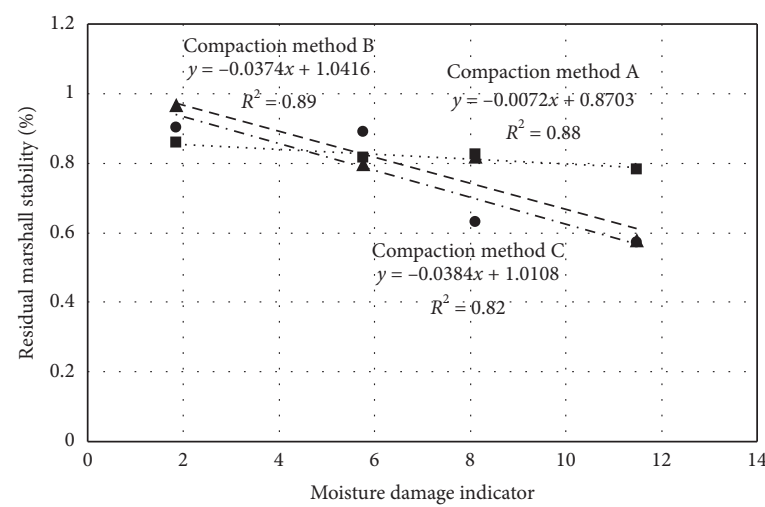

- Compaction method $\mathrm{A}$

- Compaction method $\mathrm{B}$

- Compaction method C

(a)

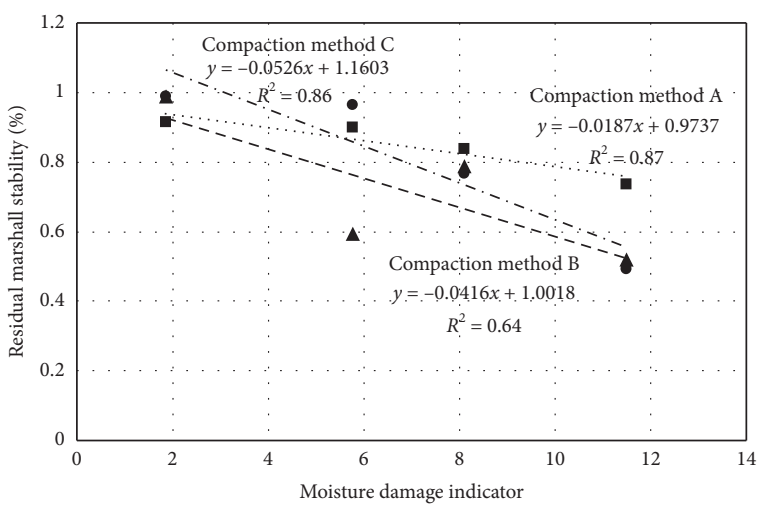

- Compaction method $\mathrm{A}$

- Compaction method B

- Compaction method C

..... Trendline-compaction method $\mathrm{A}$

- - - Trendline-compaction method B

-. - . Trendline-compaction method C

(b)

FIgURE 8: Correlation between RMS and moisture damage indicator for (a) short- and (b) long-term performance.

The results indicated that the DG CMA patching materials did not exhibit high abrasion resistance in the Cantabro test. Among OG CMA patching materials, CMA-1 exhibited the lowest Cantabro abrasion ratio, whereas CMA-2 exhibited the second highest for both short- and long-term performance. Because the asphalt film thickness is a critical factor 


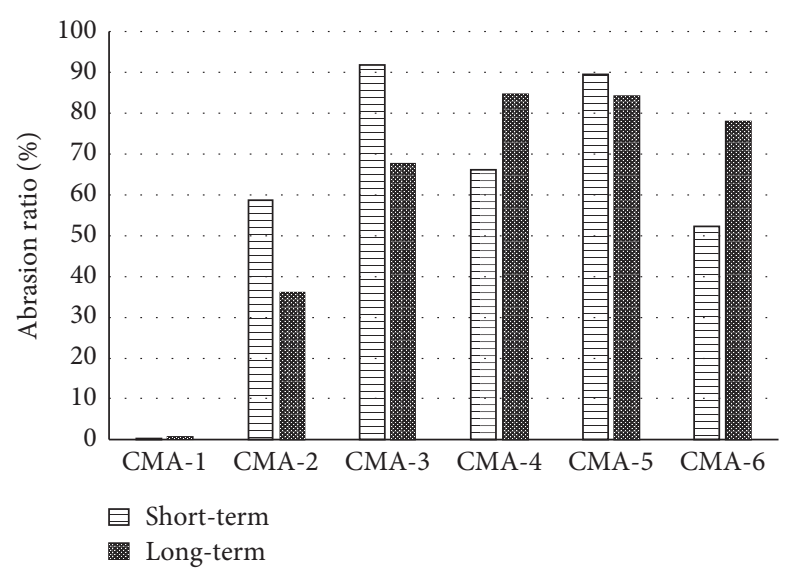

Figure 9: Abrasion ratio for short- and long-term performance.

that affects the ability of mixtures to resist abrasion, it was considered an indicator for identifying the abrasion resistance of OG CMA patching materials. The asphalt film thickness was calculated using the aggregate surface area and asphalt binder content. The surface area of aggregate can be estimated from the aggregate gradation as follows [2]:

$$
\begin{aligned}
\mathrm{SA}= & 0.205 *\left[2 * P_{\#(3 / 4)}+2 * P_{\#_{4}}+4 * P_{\# 8}+8 * P_{\# 16}\right. \\
& \left.+14 * P_{\# 30}+30 * P_{\# 50}+60 * P_{\# 100}+160 * P_{\#_{200}}\right],
\end{aligned}
$$

where SA is the surface area (in square meters) per kilogram of aggregate, $P$ is the passing aggregate (\%), and the subscript of $\mathrm{P}$ denotes the sieve size. The asphalt film thickness can be estimated using the following expression [2]:

$$
T_{F}=\left(\frac{W_{b} / G_{b}}{\mathrm{SA} * W_{a}}\right) * 1000,
$$

where $T_{F}$ is the asphalt film thickness (in micrometers), $W_{b}$ and $W_{a}$ are the weights of the asphalt binder and aggregate, respectively, and $G_{b}$ is the asphalt specific gravity. However, because the collected CMA patching materials were commercially purchased, accurate volumetric properties, specifically the effective specific gravity of aggregate, were difficult to obtain. Therefore, instead of the effective asphalt content, the residual asphalt content shown in Table 1 was used to calculate the estimated asphalt film thickness. Figure 10 depicts the correlation between the Cantabro abrasion ratio and estimated asphalt film thickness. A thicker asphalt film resulted in higher abrasion resistance; the coefficient of correlation was 0.83 and 0.88 for shortand long-term performance, respectively. These results indicated that the asphalt film thickness is highly correlated with the abrasion resistance of OG CMA patching materials.

\subsection{Cantabro Test: Moisture Damage Evaluation for Abrasion.}

To investigate the effects of moisture damage on abrasion, the Cantabro test was conducted on CMA patching materials subjected to moisture damage (i.e., curing processes $\mathrm{C} 2$ and C4). Figure 11 presents the Cantabro test results for

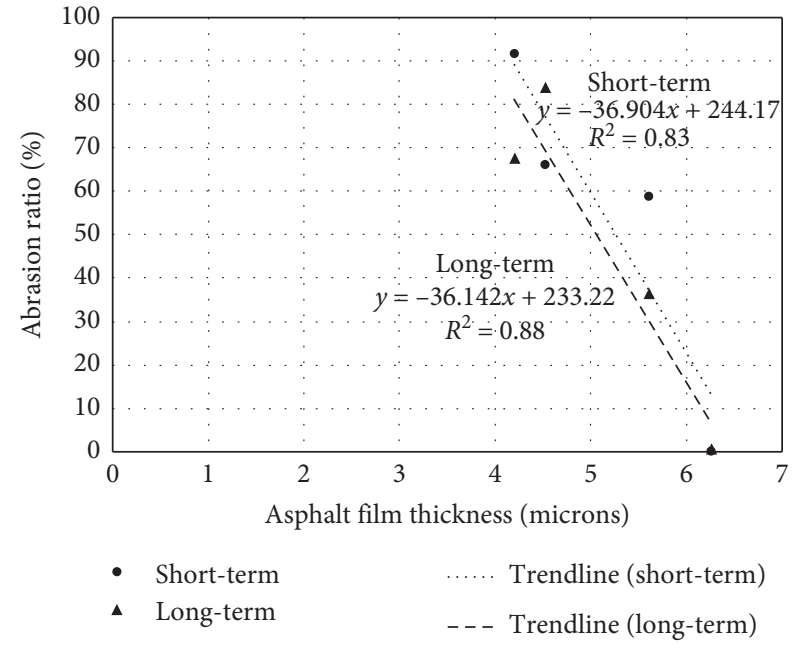

FIgURE 10: Correlation between the abrasion ratio and asphalt film thickness for short- and long-term performance.

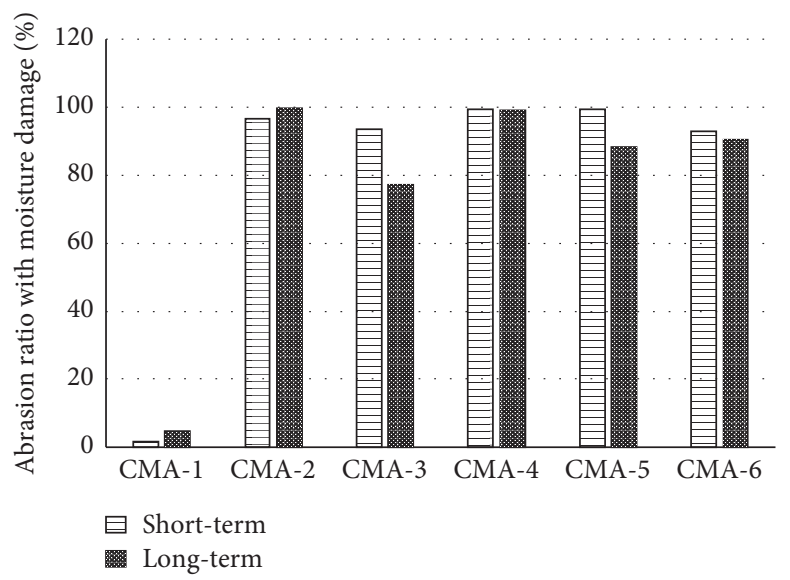

FIGURE 11: Abrasion ratio for short- and long-term performance with moisture damage.

short-term (C2) and long-term (C4) performance with moisture damage. The results revealed that the abrasion ratios were more than $80 \%$ in most of CMA patching 
materials for both short- and long-term performance, except for CMA-1. Moreover, the DG CMA patching materials did not exhibit high moisture damage resistance in the Cantabro test.

To evaluate the effect of moisture on abrasion, the loss abrasion ratio because of moisture damage was calculated by subtracting the Cantabro abrasion ratio with moisture damage from that without as follows:

$$
R_{\mathrm{imd}}=R_{\mathrm{am}}-R_{\mathrm{ad}},
$$

where $R_{\text {imd }}$ is the increase in the abrasion ratio because of moisture damage. Here, $R_{\mathrm{am}}$ is the abrasion ratio with moisture damage and $R_{\mathrm{ad}}$ is the abrasion ratio in the dry condition. Figure 12 illustrates the correlation between $R_{\text {imd }}$ and MI, as obtained in equation (3), for OG CMA patching materials. The coefficient of correlation was 0.75 and 0.68 for short- and long-term performance, respectively. These coefficients were lower than that obtained for RMS, as depicted in Figure 8. This result indicates that the effect of moisture on abrasion loss is related not only to aggregate gradation but to the combined effect of aggregate gradation and asphalt film thickness. Furthermore, in the Cantabro test, CMA-1 exhibited high moisture damage resistance, whereas CMA-2 is susceptible to moisture damage. These results concurred with the RMS results, and the proposed MI is a valid indicator to represent the moisture susceptibility for OG CMA patching materials.

\subsection{UK Wheel Tracking Test: Rutting Evaluation.} Figure 13 depicts the cycles to a rutting depth of $20 \mathrm{~mm}$ for all collected CMA patching materials. The DG CMA patching materials exhibited higher rutting resistance than the OG CMA patching materials. Moreover, among the OG CMA patching materials, CMA-1 exhibited the highest rutting resistance, which is consistent with the Marshall stability test results. Figure 14 depicts the correlation between cycles to a rutting depth of $20 \mathrm{~mm}$ and the proportion of coarse aggregate for the OG CMA patching materials. The coefficient of correlation was 0.7 , which is lower than that in the Marshall stability tests. This reduction is likely because the wheel tracking test mechanism was more complex than the Marshall stability test. Nevertheless, the proportion of coarse aggregate still can effectively indicate the rutting resistance and strength of OG CMA patching materials.

\section{Conclusions and Suggestions}

The performance and physical properties of six commercially purchased CMA patching materials-four OG and two DG mixtures-were assessed. Three sample fabrication processes were used to investigate the effect of sample fabrication conditions on the Marshall stability of CMA patching materials. Four curing processes were simulated to represent the short-term (initial stability), long-term (inservice), and moisture damage in the field. The following conclusions were derived:

(i) The compaction of CMA patching materials under heating resulted in considerably higher Marshall

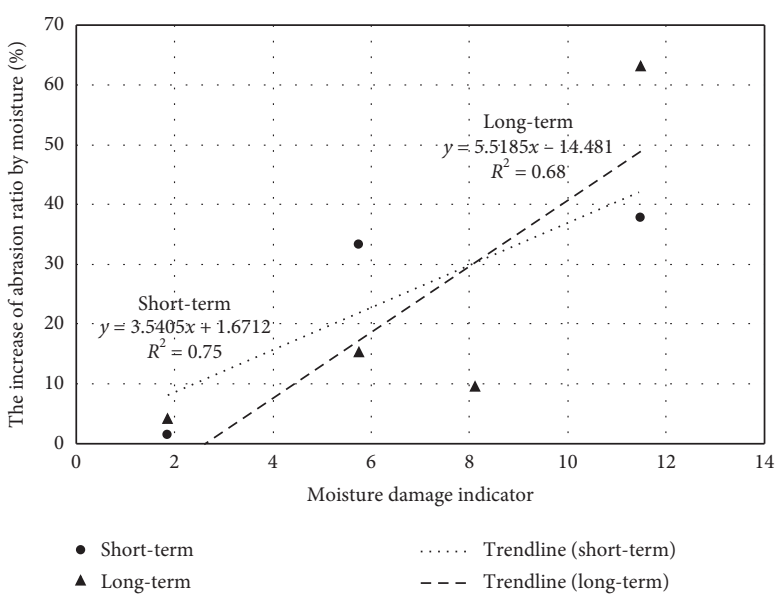

FIgURE 12: Correlation between the increase in the abrasion ratio with moisture and moisture damage indicator for short- and longterm performance.

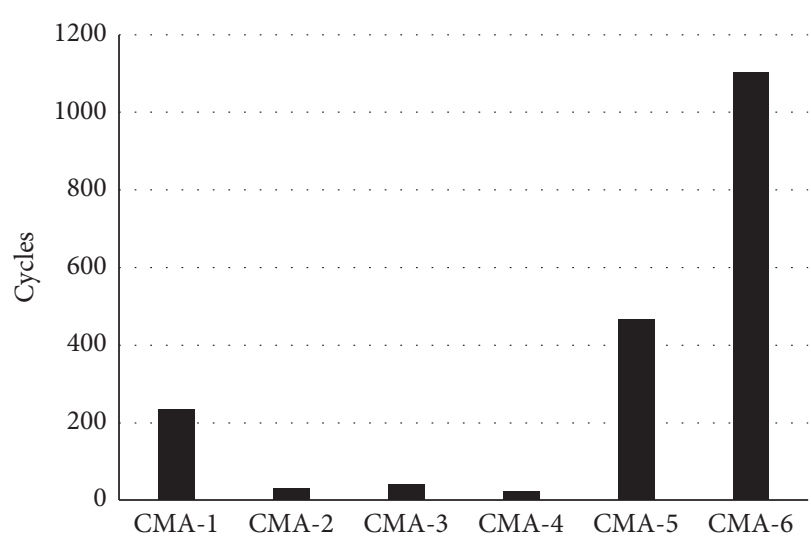

FIGURE 13: Results of UK wheel tracking test.

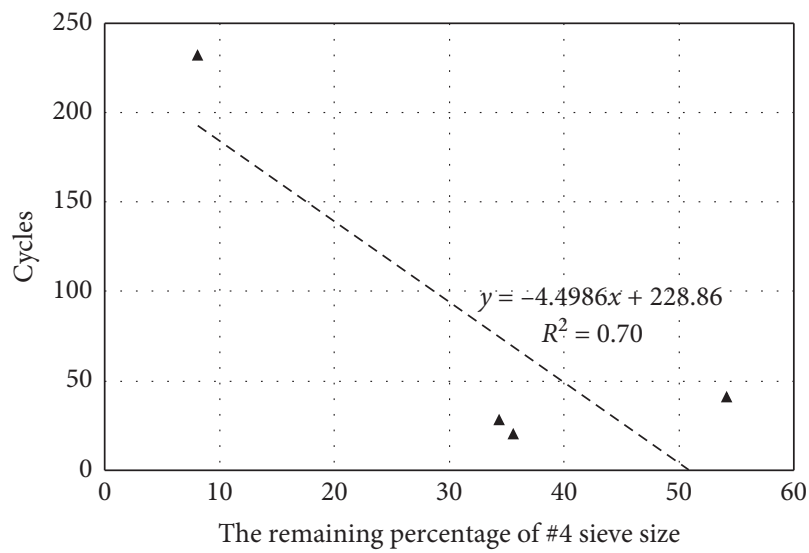

FIgURE 14: Correlation between cycles to rutting of $20 \mathrm{~mm}$ and the proportion of coarse aggregate.

stability than compaction at the ambient temperature. The compaction under heating in a laboratory thus resulted in overestimation of the field strength of CMA patching materials. 
(ii) The DG CMA patching materials exhibited higher Marshall stability than OG CMA patching materials. For OG CMA patching materials, the proportion of coarse aggregate was highly correlated to Marshall stability.

(iii) The effect of moisture damage on Marshall stability of OG CMA patching materials was highly correlated with the proposed MI. The average coefficients of correlation were 0.86 and 0.78 for short- and long-term performance, respectively.

(iv) The DG CMA patching materials did not exhibit high abrasion resistance for both short- and long-term performance. The Cantabro abrasion ratio was highly correlated with the estimated asphalt film thickness of OG CMA patching materials. The coefficients of correlation were 0.82 and 0.88 for short- and long-term performance, respectively.

(v) The correlation between the abrasion ratio because of moisture damage and MI decreased to 0.68 and 0.75 for short- and long-term performance, respectively. Thus, the effect of moisture on abrasion was related not only to MI but also to the combined effect of aggregate gradation and asphalt film thickness. Nevertheless, the proposed MI could indicate the effect of moisture on abrasion for OG CMA patching materials.

(vi) The UK wheel tracking test indicated that the DG CMA patching materials have higher rutting resistance than OG CMA patching materials, which is consistent with the Marshall stability test results. Moreover, the proportion of coarse aggregate was correlated with rutting resistance for OG CMA patching materials.

(vii) The indicators proposed in this study were only based on six ready-to-use CMA patching materials. Studies on more CMA patching materials are required to verify the correlation between the proposed indicators and resistance to moisture damage, abrasion, and rutting. Moreover, the proposed indicators should be validated by comparing the field performance of CMA patching materials.

(viii) The collected CMA patching materials in this study were produced by manufacturing companies, and this limited the evaluation of fundamental properties of materials. It is necessary to comprehensively investigate the effect of fundamental properties (e.g., asphalt binder type, the adhesion between asphalt and aggregate, and aggregate surface properties) on the performance of CMA patching materials.

(ix) The tests used in this study were experience-based tests. Further tests related to performance and mechanic tests (e.g., dynamic modulus test and Hamburg wheel tracking test) are desired.

\section{Data Availability}

The data used to support the findings of this study are included within the article.

\section{Conflicts of Interest}

The authors declare no conflicts of interest regarding the publication of this paper.

\section{Acknowledgments}

The authors thank the Ministry of Science and Technology for supporting this study.

\section{References}

[1] S.-M. Lu, C. Lu, K.-T. Tseng, F. Chen, and C.-L. Chen, "Energy-saving potential of the industrial sector of Taiwan," Renewable and Sustainable Energy Reviews, vol. 21, pp. 674683, 2013.

[2] F. L. Roberts, P. S. Kandhal, E. R. Brown, D.-Y. Lee, and T. W. Kennedy, Hot Mix Asphalt Materials, Mixture Design and Construction, National center for asphalt technology, Lanham, MD, USA, 2nd edition, 1996.

[3] Y. Yue, M. Abdelsalam, D. Luo, A. Khater, J. Musanyufu, and T. Chen, "Evaluation of the properties of asphalt mixes modified with diatomite and lignin fiber: a review," Materials, vol. 12, no. 3, pp. 400-416, 2019.

[4] Y. H. Huang, Pavement Analysis and Design, Pearson Prentice Hall, Upper Saddle River, NJ, USA, 2nd edition, 2004.

[5] A. Yilmaz and S. Sargin, "Water effect on deteriorations of asphalt pavements," Online Journal of Science Technology, vol. 2, no. 1, pp. 1-6, 2012.

[6] Y.-R. Kim, J. Zhang, and H. Ban, "Moisture damage characterization of warm-mix asphalt mixtures based on laboratory-field evaluation," Construction and Building Materials, vol. 31, pp. 204-211, 2012.

[7] C. Ling, R. Moraes, D. Swiertz, and H. Bahia, "Measuring the influence of aggregate coating on the workability and moisture susceptibility of cold-mix asphalt," Transportation Research Record: Journal of the Transportation Research Board, vol. 2372, no. 1, pp. 46-52, 2013.

[8] A. A. Munyagi, Evaluation of Cold Asphalt Patching Mixes, Ph.D. thesis, University of Stellenbosch, Stellenbosch, South Africa, 2007.

[9] M. Rezaei, L. Hashemian, A. Bayat, and B. Huculak, "Investigation of rutting resistance and moisture damage of cold asphalt mixes," Journal of Materials in Civil Engineering, vol. 29, no. 10, 2017.

[10] M. C. Liao, C. C. Luo, T. Y. Wang, and X. Xie, "Developing effective test methods for evaluating cold-mix asphalt patching materials," Journal of Materials in Civil Engineering, vol. 28, no. 10, 2016.

[11] J.-S. Chen, H.-C. Ho, M. C. Liao, M.-C. Liao, and T.-Y. Wang, "Engineering properties of asphalt concrete patching mixtures," Journal of Testing and Evaluation, vol. 44, no. 1, pp. 20140441-20140449, 2016.

[12] J. Minegishi, T. Takeda, M. Tatsushita, H. Ohki, and S. Wataya, "A study on cold mixtures for pothole repair in Tokyo," in Proceedings of 11th International Conference on Asphalt Pavements, ISAP, Nagoya, Japan, August 2010.

[13] S. Al-Busaltan, H. Al Nageim, W. Atherton, W. Atherton, and G. Sharples, "Mechanical properties of an upgrading cold-mix 
asphalt using waste materials," Journal of Materials in Civil Engineering, vol. 24, no. 12, pp. 1484-1491, 2012.

[14] L. G. Diaz, "Creep performance evaluation of cold mix asphalt patching mixes," International Journal of Pavement Research and Technology, vol. 9, no. 2, pp. 149-158, 2016.

[15] C. K. Estakhir and J. W. Button, Evaluation and Improvement of Bituminous Maintenance Mixtures, Texas Transportation Institute, Texas A\&M University, College Station, TX, USA, 1995.

[16] V. I. Rosales-Herrera, J. Prozzi, and J. A. Prozzi, Mixture Design and Performance-Based Specifications for Cold Patching Mixtures, Texas Dept. of Transportation, Austin, TX, USA, 2007.

[17] AASHTO, Standard Method of Test for Quantitative Extraction of Asphalt Binder from Hot Mix Asphalt (HMA), AASHTO T164, Washington, DC, USA, 2014.

[18] AASHTO, Standard Method of Test for Mechanical Analysis of Extracted Aggregate, AASHTO T30, Washington, DC, USA, 2015.

[19] S. S. Jhou, "Asphalt cold mix patching material of durability research," Master thesis, National Central University, Taoyuan, Taiwan, 2009.

[20] ASTM, Standard Test Method for Marshall Stability and Flow of Bituminous Mixtures, ASTM D6927, West Conshohocken, PA, USA, 2006.

[21] ASTM, Standard Practice for Open-Graded Friction Course (OGFC) Mix Design, ASTM D7064, West Conshohocken, PA, USA, 2013.

[22] BSI, Bituminous Mixtures. Test Methods for Hot Mix Asphalt. Wheel Tracking, BS EN 12697-22, London, UK, 2004.

[23] G. H. Hamedi and F. M. Nejad, "Evaluating the effect of mic design and thermodynamic parameters on moisture sensitivity of hot mix asphalt," Journal of Materials in Civil Engineering, vol. 29, no. 2, 2016.

[24] F. M. Nejad, M. Asadi, G. H. Hamedi, and M. R. Esmaeeli, "Using hydrophobic coating on aggregate surfaces to reduce moisture damage in asphalt mixture," Journal of Materials in Civil Engineering, vol. 30, no. 10, 2018.

[25] F. M. Nejad, M. Arabani, G. H. Hamedi, and A. R. Azarhoosh, "Influence of using polymeric aggregate treatment on moisture damage in hot mix asphalt," Construction and Building Materials, vol. 47, pp. 1523-1527, 2013.

[26] J. Howson, A. Bhasin, E. Masad, R. Lytton, and D. Little, Development of a Database or Surface Energy of Aggregate and Asphalt Binders, Texas Transportation Institute, College Station, TX, USA, 2009.

[27] G. H. Hamedi and F. Moghadas Nejad, "Use of aggregate nanocoating to decrease moisture damage of hot mix asphalt," Road Materials and Pavement Design, vol. 17, no. 1, pp. 32-51, 2015.

[28] A. Varveri, S. Avgerinopoulos, C. Kasbergen, A. Scarpas, and A. Collop, "Influence of air void content on moisture damage susceptibility of asphalt mixtures," Transportation Research Record: Journal of the Transportation Research Board, vol. 2446, no. 1, pp. 8-16, 2014.

[29] C. Gorkem and B. Sengoz, "Predicting stripping and moisture induced damage of asphalt concrete prepared with polymer modified bitumen and hydrated lime," Construction and Building Materials, vol. 23, no. 6, pp. 2227-2236, 2009. 